TITLE:

\title{
Modeling the trajectory of window flames with regard to flow attachment to the adjacent wall
}

\author{
AUTHOR(S): \\ HIMOTO, Keisuke; TSUCHIHASHI, Tsuneto; \\ TANAKA, Yoshiaki; TANAKA, Takeyoshi
}

\section{CITATION:}

HIMOTO, Keisuke ... [et al]. Modeling the trajectory of window flames with regard to flow attachment to the adjacent wall. Fire Safety Journal 2009, 44(2): 250-258

\section{ISSUE DATE:}

2009-02

URL:

http://hdl.handle.net/2433/93451

\section{RIGHT:}

Copyright (C) 2008 Elsevier Ltd; This is not the published version. Please cite only the published version.; この論文は出版社版でありません。引 用の際には出版社版をご確認ご利用ください。 


\section{Modeling Trajectory of Window Flame with regard to The Flow Attachment to The Adjacent Wall}

Keisuke HIMOTO (corresponding author)

Pioneering Research Unit for Next Generation, Kyoto Univ.

Gokasho, Uji, Kyoto 611-0011, Japan

e-mail keisuke.himoto@kupru.iae.kyoto-u.ac.jp

tel. $\quad+81-774-38-4481$

fax $+81-774-38-4039$

Tsuneto TSUCHIHASHI, and Yoshiaki TANAKA

General Building Research Corp. of Japan

Fujishirodai 5-8-1, Suita, Osaka 565-0873, Japan

Takeyoshi TANAKA

Disaster Prevention Research Institute, Kyoto Univ.

Gokasho, Uji, Kyoto 611-0011, Japan

\section{ABSTRACT}

A model for predicting the trajectory of window flame ejected from a fire compartment was formulated incorporating the effect of wall above the opening. Based on the observation in the 
reduced scale experiments, window flames were divided into following categories with regard to its trajectory configuration: the flow which ascends almost vertically up after ejection maintaining a certain separation from the wall; and the flow which ascends upward after ejection and gradually approaches to the wall in the downstream. In the model, trajectories of these flows were approximated by cubic polynomials whose coefficients were given as functions of a dimensionless parameter $F^{*}$. The parameter $F^{*}$ was derived from the conservation equation of momentum which incorporates the effect of pressure gradient across the ascending flows. Critical condition for the occurrence of flow attachment was described as a proportion of the maximum separation from the wall versus the opening width. Trajectories predicted by the proposed model were then compared with the measurement data which indicated reasonable agreements.

Keywords: fire spread; window flame; trajectory; reduced-scale experiment; scale model

\section{INTRODUCTION}

When fire inside a compartment enlarges and becomes to be at its most vigorous stage, flame will be ejected from the opening after the failure of window glass. Heat flux transferred from the window flame yields ignition of combustibles stored inside the upper floors as well as those 
in the adjacent buildings. As it is one of the most important contributing factors for fire spread between rooms, window flame has received considerable attention and there are already several models available. In such models, most substantial effort has been devoted on temperature rise prediction, as it is closely related to the hazard evaluation of window flame [1-11]. The achieved knowledge is widely applied to the practice of building design in which it provides quantitative measures for fire safety evaluation. However, most of the existing models predict temperature rise along trajectory, i.e., the curve sequentially connecting points of the largest temperature rise at each height. In other words, the hazard of a window flame cannot be quantified without knowing its trajectory configuration, as the rate of heat transfer from the window flame to adjacent combustible needs to be evaluated in terms of the separation between them.

In contrast with the substantial progress in the temperature rise prediction methodology, there is not much work done on the measures of predicting trajectory configuration. One of the few examples is the model proposed by Yokoi [1] which is given by,

$$
\frac{Z}{H-Z_{N}}=\frac{1}{9 \beta T_{\infty}}\left\{\left(\frac{x+X_{0}}{H-Z_{N}}\right)^{3 / 2}-\left(\frac{X_{0}}{H-Z_{N}}\right)^{3 / 2}\right\}^{2} /\left(\frac{X_{0}}{H-Z_{N}}\right)
$$

where $x$ is the separation from the wall, $z$ is the vertical height, $x_{0}$ is the depth of the virtual origin, $H$ is the height of the opening, $Z_{N}$ is the height of the neutral plane, and $\beta$ is the thermal expansion coefficient. The model describes the trajectory configuration with the 
cubic function, in which the flow is initially ejected in the horizontal direction and gains vertical momentum due to buoyancy in the downstream. It shows reasonable agreement with the experimental data when no interaction is involved between the window flame and the wall above the opening [1]. However, this is not a common assumption for buildings in general. One of the typical consequences of the interaction is the flow attachment to the wall above the opening. This is often observed when the width of the opening is large. In such a condition, pressure of the space sandwiched between the wall and the window flame decreases, as the flame entrains air from the sandwiched space, while supply of air to the sandwiched space is restricted due to the presence of the wall $[1,11]$. This yields the pressure gradient across the flow, and as a result, draws the window flame to the wall [1]. In order to characterize such behavior, a trajectory model which incorporates the effect of the wall-flame interaction was derived, and was verified with the data obtained in the previous experiment [11].

\section{TRAJECTORY OF WINDOW FLAME}

\subsection{Difference in Pressure between Wall-side and Open-side of The Trajectory}

The first parameter which needs to be modeled is the pressure difference $\Delta p$, which yields the attachment of window flame to the wall. Figure 1 shows the horizontal section of window flame in relation to the adjacent wall. For the convenience of description, a coordinate system is 
introduced: the origin is at the point of the maximum temperature rise in the window plane;

$x$-axis is perpendicular to the window plane; $z$-axis is vertically upward; and $y$-axis

perpendicular to the other axes. We now assume a thin layer element in $z$-direction which is divided into three regions of different characteristics: 1) buoyancy-driven region that constitute main body of the window flame (flame region); 2) region sandwiched between the flame region and the wall (by-wall region); and 3) the rest of the space which may be considered as the ambient environment (ambient region). Moreover, the entrainment of ambient air into the window flame is assumed to be induced by the pressure difference between the flame region and the other regions. As the flame region entrains air from the other regions, pressure inside the flame region $p_{m}$ should be the minimum among the three regions. However, as the entrainment of air from the by-wall region tends to be restricted due to the lack of the space, while it is not from the ambient region, following relation generally applies,

$$
p_{m}<p_{\infty, W} \leq p_{\infty}
$$

where $p_{\infty, W}$ and $p_{\infty}$ are the characteristic pressure of the by-wall region and the ambient region, respectively. The flow attachment is yielded by the pressure difference between the by-wall region and the ambient region $\Delta p$, which is given by,

$$
\Delta p=p_{\infty}-p_{\infty, W}
$$


When the ejected flow is incompressible and ascends almost vertically upward, mass transfer between the three regions is evaluated by the Bernoulli's theory. The pressure difference between the regions is equivalent to the flow momentum across the boundary, i.e.,

$p_{\infty}-p_{m} \sim \frac{1}{2} \rho_{\infty} U_{E}^{2}, \quad p_{\infty, W}-p_{m} \sim \frac{1}{2} \rho_{\infty} U_{E, W}^{2}, \quad p_{\infty}-p_{\infty, W} \sim \frac{1}{2} \rho_{\infty} V_{E, W}^{2}$

where $U_{E}$ is the characteristic flow velocity from the ambient region to the flame region, $U_{E, W}$ is that from the by-wall region to the flame region, $V_{E, W}$ is that from the ambient region to the by-wall region, and $\rho_{\infty}$ is the gas density in the ambient region and the by-wall region.

Eliminating the pressure terms from Eqn.(4), we obtain,

$U_{E}^{2} \sim U_{E, W}^{2}+V_{E, W}^{2}$

Assuming that the effect of flow-wall interaction on the entraining flow velocity $U_{E}$ is minor, and the entrainment rate is proportional to the trajectory velocity $u_{m}$, which is a common assumption for the classical models of vertically ascending fire plumes [12], we obtain,

$U_{E}=\alpha u_{m}$

Approximating the shapes of the flame region (abcd) and that of the by-wall region (a'b'c'd') by rectangles, and neglecting overall mass transfer in $z$-direction for the by-wall region, then the mass conservation equation for the by-wall region can be expressed as, 
$D_{y} U_{E, W}=2 D_{x} V_{E, W}$

where $D_{x}$ is the characteristic $x$-wise length of the by-wall region, $D_{y}$ is that of $y$-wise length. By solving Eqns.(5)-(7) simultaneously, flow velocities at the boundaries $U_{E, W}$ and $V_{E, W}$ are obtained as follow,

$U_{E, W} \sim \frac{\alpha u_{m}}{\sqrt{1+\left(D_{y} / 2 D_{x}\right)^{2}}}, \quad V_{E, W} \sim \frac{\alpha u_{m}}{\sqrt{1+\left(2 D_{x} / D_{y}\right)^{2}}}$

This equation shows that the magnitudes of the boundary flow velocities $U_{E, W}$ and $V_{E, W}$ are dependent on the aspect ratio of the by-wall region. That is, when the lengths of the air entrainment boundaries a'd' and b'c' are short ( $D_{x}<<D_{y}$ ), the boundary flow velocities become $U_{E, W}<<V_{E, W}$. Whereas when the lengths of the air entraining boundaries a'd' and b'c' are large ( $D_{x}>>D_{y}$ ), the boundary flow velocities become $U_{E, W}>>V_{E, W}$. As for the latter case, the entrainment rates become congruent regardless of their position, i.e., $U_{E} \cong U_{E, W}$, as abundant air is supplied into the by-wall region. This relation, $U_{E} \cong U_{E, W}$, is commonly observed in the vertically ascending fire plumes independent of the wall interference.

Finally, by substituting the boundary flow velocity $V_{E, W}$ into Eqns.(3) and (4), then the pressure difference which yields the attachment of the window flame is obtained as follows,

$$
\Delta p \sim \frac{1}{2} \rho_{\infty}\left(\alpha u_{m}\right)^{2} \cdot\left[\frac{1}{1+\left(2 D_{x} / D_{y}\right)^{2}}\right]
$$

Now we assume that the characteristic $x$-wise length of the by-wall region $D_{x}$ can be 
approximated by the distance of the trajectory from the wall surface, i.e.,

$D_{x} \cong x$

In addition to this, accounting that the spreading width of window flame in the direction

perpendicular to the trajectory is generally short $[4,9,11]$, the characteristic $y$-wise length of

the by-wall region is approximated by the opening width,

$D_{y} \cong B$

By substituting Eqns.(10) and (11) into Eqn.(9), then the pressure difference $\Delta p$ can be

rewritten as follows,

$\Delta p \sim \frac{1}{2} \rho_{\infty}\left(\alpha u_{m}\right)^{2} \cdot\left[\frac{1}{1+(2 x / B)^{2}}\right]$

This equation shows that when the window flame gets well apart from the wall surface $(x>>B)$, then the pressure difference $\Delta p$ which draws the flame to the wall becomes negligible $(\Delta p \rightarrow 0)$.

\subsection{Modeling Trajectory of Window Flame}

The approach we take here for the trajectory modeling is to approximate it with a curve and describe its consisting coefficients with the pressure difference $\Delta p$ in Eqn.(12). According to the observation in the previous experiment [11], trajectories of window flame can be divided 
into two types: flow that ascends almost vertically maintaining certain separation from the wall;

and flow that ascends upward after ejection and attach to the wall in the downstream. Firstly, we approximate the latter by the following $(\mathrm{n}+2)$-variable polynominal,

$\frac{X}{L_{E}}= \begin{cases}C_{n+2}\left(\frac{Z}{H_{A}}\right)^{n+2}+C_{n+1}\left(\frac{Z}{H_{A}}\right)^{n+1}+C_{n}\left(\frac{Z}{H_{A}}\right)^{n}+C_{0} & \left(0 \leq Z \leq H_{A}\right) \\ 0 & \left(H_{A} \leq Z\right)\end{cases}$

where $L_{E}$ is the separation between the equilibrium point and the wall surface, $H_{A}$ is the vertical height of the attachment point, and $c_{0}, c_{n}, c_{n+1}, c_{n+2}$ are the coefficients. The equilibrium point is the position at which the flow momentum in the horizontal direction becomes zero, and the attachment point is the position at which the trajectory touches the wall surface. In Eqn.(13), the window flame ascends vertically upward along the wall surface after the attachment. Note that the flow velocity becomes zero on the wall surface, and the trajectory does not attach to the wall in a strict sense. However, upon the basis of the observed trajectory configurations in the experiments [11], this approximation is reasonable in a practical sense.

Applying the boundary conditions for both the ejection point and the attachment point,

$$
\left.\begin{array}{ll}
Z=0: \quad x=0 & \text { (ejection point) } \\
Z=H_{A}: x=0, \frac{\partial x}{\partial z}=0 & (\text { attachment point })
\end{array}\right\}
$$

into Eqn.(13), the polynominal is transformed into, 
$\frac{x}{L_{E}}= \begin{cases}c\left(\frac{z}{H_{A}}\right)^{n}\left(1-\frac{z}{H_{A}}\right)^{2} & \left(0 \leq z \leq H_{A}\right) \\ 0 & \left(H_{A} \leq z\right)\end{cases}$

in which the coefficient $c_{n+2}$ was substituted with another coefficient $c$. By differentiating

Eqn.(15) with respect to $z$, then the gradient of the trajectory within the range $0 \leq z \leq H_{A}$ is

given as follows,

$\frac{\partial x}{\partial z}=c(n+2) \frac{L_{E}}{H_{A}}\left(\frac{z}{H_{A}}\right)^{n-1}\left(1-\frac{z}{H_{A}}\right)\left(\frac{n}{n+2}-\frac{z}{H_{A}}\right)$

The left hand side of the equation becomes zero, i.e., the trajectory becomes parallel to the wall surface, either when $z=n /(n+2) \cdot H_{A} \equiv H_{E}$ or when $z=H_{A}$. These $z$ 's represent nothing but the heights of the equilibrium point $H_{E}$ and that of the attachment point $H_{A}$, respectively. Eqn.(16) also shows that the height of the equilibrium point $H_{E}$ is proportional to both the height of the attachment point $H_{A}$ and the parameter $n$. The value of $n$ is adjusted so that the model reproduces the experimental data well. Out of the 20 test cases carried out, $H_{A}$ and $H_{E}$ were discernible in 7 cases [11]. The relationship between $H_{A}$ and $H_{E}$ is shown in Fig. 3 with lines at different $n$ 's. Although there is certain variation between the plots, $n=1$ is adopted referring to this results. In this case, the trajectory is approximated by a cubic function, and the height of the equilibrium point is given by,

$H_{E}=\frac{H_{A}}{3} \quad(n=1)$

Additionally, as the trajectory passes through the equilibrium point ( $x=L_{E}$ when $z=H_{A} / 3$ ), 
the value of the remaining coefficient $c$ is obtained from Eqn.(15),

$c=\frac{27}{4}$

As a result of all these discussion, we obtain expression for the attaching flame trajectory,

Attaching flame: $\frac{x}{L_{E}}= \begin{cases}\frac{27}{4}\left(\frac{z}{H_{A}}\right)\left(1-\frac{z}{H_{A}}\right)^{2} & \left(0 \leq z \leq H_{A}\right) \\ 0 & \left(H_{A} \leq z\right)\end{cases}$

As for the trajectory of non-attaching flame, we assume that the curved configuration of the trajectory from the venting point to the equilibrium point $\left(x=L_{E}, \quad z=H_{E}\right)$ is brought by the same mechanism as that of the attaching flame. As a result, the trajectory of non-attaching flame takes the identical configuration to that of Eqn.(19) before it reaches the equilibrium point. In the subsequent phase, the trajectory is drawn towards the wall due to $\Delta p$ for the attaching flame, while the effect of $\Delta p$ can be neglected for the non-attaching flame. As for the non-attaching flame, flow momentum in the horizontal direction is balanced out with the work done by $\Delta p$ before it reaches the equilibrium point. Thus, the flame rises vertically upward in the downstream as,

Non-attaching flame: $\quad \frac{x}{L_{E}}= \begin{cases}\frac{27}{4}\left(\frac{z}{H_{A}}\right)\left(1-\frac{z}{H_{A}}\right)^{2} & \left(0 \leq z \leq H_{E}\right) \\ 1 & \left(H_{E} \leq z\right)\end{cases}$

\subsection{Equivalent Point and Attachment Point}


Configuration of the trajectory can be estimated with the derived Eqns.(19) and (20).

However, the expressions have no physical background at this moment except for that the boundary conditions. Thus the separation of the equilibrium point $L_{E}$ and the height of the attachment point $H_{A}$ which are involved in these equations, are modeled in order to fulfill the momentum balance in the horizontal $(x)$ direction. For simplicity, we assume that the momentum in $x$-direction decreases mainly due to the pressure difference,

$\frac{d}{d x}\left(b u_{m}^{2}\right)=-\frac{\Delta p}{\rho_{\infty}}$

where $b$ is the characteristic width of the window flame when the velocity in $x$-direction takes the Gaussian profile. Based upon the experimental observation [11], we assumed that the virtual heat source is just beneath the upper edge of the window, and has elongate shape in $y$-direction. In other words, line heat source assumption is invoked, and profiles of temperature and velocity in the $y$-direction are neglected. Substituting the pressure difference $\Delta p$ into Eqn.(21), we obtain,

$$
\frac{d}{d x}\left(b u_{m}^{2}\right) \sim-\frac{\left(\alpha u_{m}\right)^{2}}{2} \cdot\left[\frac{1}{1+(2 x / B)^{2}}\right]
$$

As defined previously, the equilibrium point $\left(x=L_{E}, \quad z=H_{E}\right)$ is the position at which the velocity in $x$-direction becomes zero. In other words, it is the point at which the momentum in the horizontal direction $\left(\propto u_{0}^{2}\left(H-Z_{N}\right)\right)$ balances out with the work done by the pressure. Thus, 
by integrating Eqn.(22) with regard to $x$,

$\phi_{1} \cdot u_{0}^{2}\left(H-Z_{N}\right) \sim \int_{0}^{L_{E}}-\frac{\left(\alpha u_{m}\right)^{2}}{2}\left[\frac{1}{1+(2 x / B)^{2}}\right] d x$

where $\phi_{1}$ is the coefficient, and $u_{0}$ is the maximum venting velocity at the window. Among the terms in Eqn. (23), the trajectory velocity $u_{m}$ can be break down into fundamental parameters by incorporating the results of the dimensional analysis [11]. Although the window flame can be divided into either the flaming regime or the plume regime, we adopt the results for the plume regime in order to derive an uninterrupted formulation. Thus the velocity along the trajectory is expressed as [11],

$u_{m}=\phi_{2} \cdot \sqrt{g\left(H-Z_{N}\right)} Q^{\prime+1 / 3}$

where $\phi_{2}$ is the coefficient, and $Q^{\prime *}$ is the dimensionless heat release rate appropriate for the line heat source flame,

$$
Q^{\prime *} \equiv \frac{\dot{Q}}{\rho_{\infty} c_{P} T_{\infty} g^{1 / 2} B\left(H-Z_{N}\right)^{3 / 2}}
$$

where $\dot{Q}$ is the virtual heat release rate of the window flame, $c_{p}$ is the specific heat of gas, $T_{\infty}$ is the ambient temperature, and $g$ is the acceleration due to gravity. Now substituting Eqn.(24) into (23), and excluding parameters independent of $x$ out of the integral, we obtain, 


$$
\begin{aligned}
\phi_{1} \cdot u_{0}^{2} \sim-\frac{\alpha^{2} \phi_{2}^{2}}{2} \cdot g Q^{\prime 2 / 2 / 3} \int_{0}^{L_{E}} \frac{d x}{1+(2 x / B)^{2}} \\
=-\frac{\alpha^{2} \phi_{2}^{2}}{2} \cdot g Q^{\prime 2 / 3 / 3} \cdot \frac{B}{2} \tan ^{-1}\left(\frac{2 L_{E}}{B}\right)
\end{aligned}
$$

After further manipulation, the separation of the equilibrium point from the wall surface $L_{E}$ is given by,

$$
\frac{L_{E}}{B} \sim \frac{1}{2} \tan \left(\frac{4 \phi_{1}}{\alpha^{2} \phi_{2}^{2}} \cdot F^{*}\right)
$$

where $F^{*}$ is another dimensionless parameter which is defined as,

$$
F^{*} \equiv\left(\frac{u_{0}}{\sqrt{g B}}\right)^{2}\left(\frac{\dot{Q}}{\rho_{\infty} c_{P} T_{\infty} g^{1 / 2} B\left(H-Z_{N}\right)^{3 / 2}}\right)^{-2 / 3}
$$

Equation (27) describes $L_{E}$ as a tangent function of the parameter $F^{*}$. However, there is a restriction with this expression that the parameter of the tangent function must be within the range of $-\pi / 2 \sim \pi / 2$. This restriction is attributed to the assumptions adopted in the derivation process and needs to be excluded for the use in practice. Note that when the parameter of the tangent function is well below 1, Eqn.(27) can be approximated by,

$$
\frac{L_{E}}{B} \sim \frac{1}{2}\left(\frac{4 \phi_{1}}{\alpha^{2} \phi_{2}^{2}} \cdot F^{*}\right)=\phi \cdot F^{*}
$$

where $\phi$ is the substitute of the other coefficients. Although the coverage of this relationship is nominally limited, we assume that it is applicable to the cases in general. Regardless of thisapproximation, the dimensional relationship between parameters in Eqn.(29) correspond 
with those of the original relationship in Eqn.(27).

Given that the ascending velocity of the flow $u_{m}$ is constant in the plume regime (Eqn.(24)), the height of the attachment point $H_{E}$ is approximately proportional to the separation in the $x$-direction $L_{E}$. In addition to this, as the height of the attachment point $H_{A}$ is proportional to that of the equilibrium point $H_{E}$ (Eqn.(17)), then $H_{A}$ also becomes proportional to $L_{E}$, $\frac{H_{A}}{B} \propto \frac{L_{E}}{B} \sim \phi \cdot F^{*}$

\section{VERIFICATION OF THE MODEL}

\subsection{The Equilibrium Point and The Attachment Point}

The observed trajectory data in the experiment were correlated with Eqns.(29) and (30). The results are shown in Fig.4, where the data of the attaching flames are indicated with solid symbols and the rest with open symbols. Regression lines for both the separation between the equilibrium point and the wall surface $L_{E}$, and the height of the attachment point $H_{A}$ were obtained by the least-square method, respectively as follow,

$$
\begin{aligned}
& \frac{L_{E}}{B}=0.21 F^{*} \quad(r=0.96) \\
& \frac{H_{A}}{B}=1.33 F^{*} \quad(r=0.90)
\end{aligned}
$$

Substituting these equations in to Eqns.(19), (20), expressions for the trajectory configuration 
are derived as,
Attaching flame:
$X^{*}=\left\{\begin{array}{l}0.603 Z^{*}\left(1.33-Z^{*}\right)^{2} \\ 0\end{array}\right.$
$\left(0 \leq Z^{*} \leq 1.33\right)$
$\left(1.33 \leq Z^{*}\right)$
Non-attaching flame:
$X^{*}=\left\{\begin{array}{l}0.603 Z^{*}\left(1.33-Z^{*}\right)^{2} \\ 0.21\end{array}\right.$
$\left(0 \leq Z^{*} \leq 0.44\right)$
$\left(0.44 \leq Z^{*}\right)$

where $X^{*}$ and $Z^{*}$ are the dimensionless parameter which is defined as follow, respectively,

$$
X^{*} \equiv \frac{X}{B} \frac{1}{F^{*}}, \quad Z^{*} \equiv \frac{Z}{B} \frac{1}{F^{*}}
$$

Prior to selecting the expression for the trajectory configuration either from Eqns.(33) or (34),

critical condition for the flow attachment needs to be determined. Note from Eqn.(12),

magnitude of the pressure difference $\Delta p$ which draws the plume towards the wall, depends on the ratio of the flame-wall separation to the opening width $x / B$. However, as the numerator $x$ is a variable number, we adopt $L_{E} / B$ as the alternative to $x / B$. From Fig.4, we can roughly deduce the critical condition for the flow attachment, as follows,

$\frac{L_{E}}{B}=0.7(-)$

below which the window flame does attach to the wall surface. In order to organize the derived equations, flowchart which shows the calculation process of the trajectory configuration is shown in Fig.5.

\subsection{Comparison of The Trajectory}


As for the model verification, two different ventilation conditions (with and without the

mechanical ventilation), at five different opening configurations were picked out as targets

(Figs.6-10). Mechanical ventilation was applied to the compartment in order to simulate

pressure elevation inside the fire compartment which may take place under the effect of external wind. Figures of series (A) show the results for the mechanically ventilated conditions

( $\dot{m}_{0}=0 \mathrm{~kg} / \mathrm{s}$ ), and those of series (B) show the results for the no mechanical ventilation ( $\dot{m}_{0}>0 \mathrm{~kg} / \mathrm{s}$ ). Figures also show the results of the model proposed by Yokoi (Eqn.(1)), which are shown with dotted lines. In fact, neither the models of the present nor Yokoi involves the effect of ventilation explicitly. However, it is implicitly reflected within the equations as the height of neutral plane $Z_{N}$ changes along with the compartment pressurization.

The results by the present model show reasonable agreements with the experimental data, in which the values of $L_{x}$ and $H_{z}$ were predicted with fair accuracy. The present model also predicted occurrence of the attachment adequately in most of the cases, whereas the Yokoi's model disregards the attachment. However, the present model showed erroneous prediction in certain conditions. Especially, in Figs. 7(A) and 8(B), the discrepancy was significant from the experimental data, as the trajectory configuration was calculated under the wrong assumption that the flow attachment does occur. With the relationship in Eqn.(31), the critical condition for the occurrence of flow attachment (Eqn.(36)) can be translated into, 
$F^{*}=3.33(-)$

While, the values of the governing dimensionless number $F^{*}$ were 2.74(-) for Fig.7(A), and 1.76(-) for Fig.8(B), respectively. These were not necessarily close to the critical value 3.33(-), and it may not be taken as they were within the margin of error. Possible causes for the lack of accuracy may be attributed to some sort of inadequacy in the model assumption. More specifically, they are: (1) incompressibility of the flow; (2) symmetry of temperature and velocity profiles along the trajectory; (3) division of horizontal plane into three regions of different characteristics. The assumption (1) is often invoked in the classical plume modeling and its legitimacy is widely approved. However, as drop of the pressure inside the flame region due to volume expansion yields the increase in the rate of air entrainment, it seems fair to expect that this intrinsic flow characteristic influences the attachment behavior. For the assumption (2), it is not always adequate to assume the symmetrical profile for window flames, especially for the attaching flames. When the profiles are asymmetrical, the rate of air entrainment also becomes asymmetrical. This may bring some inconsistency with the homogeneity assumption on combustion and the pressure formation inside the segmented regions. On the assumption (3), we assumed that the attachment of window flame induced by the pressure difference between three regions. However, adequacy of this assumption is not fully validated with quantitative experimental data. In order to verify, measurement of three dimensional profiles of the flow 
velocity as well as the static pressure will be needed.

\section{CONCLUSIONS}

In this paper, a trajectory of window flame was modeled with special emphasis on the flow attachment to the wall. In the model, the flow attachment was assumed to be induced by the pressure difference between the by-wall region and the ambient region. The pressure difference $\Delta p$ was modeled by considering the exchange of mass and momentum between the segmented regions around the window flame. The configuration of the flame trajectory was approximated by a cubic polynominal, which showed the best agreement to the experimental data. The parameters involved within the approximated curve were determined in order to comply with the conservation equation of momentum, in which the modeled pressure difference $\Delta p$ was incorporated.

The results of the model prediction were reasonably comparable to the experimental data.

However, the model gave erroneous judgment for the occurrence of flow attachment in certain conditions. As a result, trajectory configurations for these conditions were not even close to the observed ones. The reason for this discrepancy is considered due to the lack of accuracy in the governing dimensionless parameter $F^{*}$. For the further model refinement, we need discussions on following issues which are invoked as assumptions in the model: (1) incompressibility of the 
flow; (2) symmetry of temperature and velocity profiles along the trajectory; (3) division of horizontal plane into three distinct regions.

\section{REFERENCES}

1. Yokoi S. Study on the prevention of fire-spread caused by hot upward current. Report of the Building Research Institute, No.34. 1960.

2. Thomas PH, Law M. The projection of flames from burning buildings. Fire Research Note, No.921. 1972

3. Oleszkiewicz I. Fire exposure to exterior walls and flame spread on combustible cladding.

Fire Technology 1990; 26(4): 357-375.

4. Sugawa O, Momita D, Takahashi W. Flow behavior of ejected fire flame/plume from an opening effected by external side wind. Proc. 5th Symp., pp.249-260. International Association for Fire Safety Science, 1997.

5. Poreh M, Morgan HP, Marshall NR, Harrison R. Entrainment by two-dimensional spill plume. Fire Safety J 1998; 30: 1-19.

6. Thomas PH, Morgan HP, Marshall N. The spill smoke in smoke control design. Fire Safety J 1998; 30: 21-46.

7. Klopovic S, Turan OF. Flames venting externally during full-scale flashover fires: two sample 
ventilation cases. Fire Safety J 1998; 31: 117-142.

8. Yamaguchi J, Iwai Y, Tanaka T, Harada K, Ohmiya Y, Wakamatsu T. Applicability of nondimensional temperature for scaling the temperatures of window jet plume. $J$ Architectural Planning and Environmental Engineering 1998; 513: 1-7 (in Japanese).

9. Ohmiya Y, Hori Y. Properties of external flame taking into consideration excess fuel gas ejected from fire compartment. J Architectural Planning and Environmental Engineering, 2001; 546: 1-8 (in Japanese).

10. Yamada T, Takanashi K, Yanai E, Suzuki T, Sekizawa A. An experimental study of ejected flames and combustion efficiency. Proc. $7^{\text {th }}$ Int. Symp., pp.903-914. International Association for Fire Safety Science, 2002.

11. Himoto K, Tsuchihashi T, Tanaka Y, Tanaka T. Modeling thermal behaviors of window flame ejected from a fire compartment. Fire Safety Journal (submitted).

12. Morton BR, Taylor GI, TurnerJS. Turbulent gravitational convection from maintained and instantaneous sources. Proc. Royal Society 1956; 234A: 1-23.

\section{NOMENCLATURE}

\section{Alphabets}

A : :Area of the opening $\left(\mathrm{m}^{2}\right)$ 
$b \quad$ :Half width of the flow velocity (m)

B :Horizontal width of the opening (m)

$c_{P} \quad:$ Heat capacity of the flow $(\mathrm{kJ} /(\mathrm{kgK}))$

$D_{x} \quad$ :Length of the $x$-wise direction of the wall region (m)

$D_{y} \quad$ :Length of the $y$-wise direction of the wall region (m)

$F^{*} \quad$ :Dimensionless parameter defined in Eqn.(28) (-)

$g \quad$ :Acceleration due to gravity $\left(\mathrm{m} / \mathrm{s}^{2}\right)$

$H \quad$ :Height of the opening (m)

$H_{A} \quad$ :Height of the attachment point (m)

$H_{E} \quad$ :Height of the equilibrium point (m)

$L_{E} \quad$ :Separation between the attachment point and the wall (m)

$\dot{m}_{0} \quad$ :Air supply rate $(\mathrm{kg} / \mathrm{s})$

$p_{m} \quad$ :Pressure of the flame region $(\mathrm{Pa})$

$p_{\infty} \quad$ :Pressure of the ambient region $(\mathrm{Pa})$

$p_{\infty, W} \quad:$ Pressure of the wall region $(\mathrm{Pa})$

$\Delta p \quad$ :Pressure gradient across the current $\left(p_{\infty}-p_{\infty, W}\right)(\mathrm{Pa})$

$\dot{Q} \quad$ :Apparent heat release rate of the window flame $(\mathrm{kW})$

$Q^{\prime *} \quad$ :Dimensionless heat release rate (-) 
$r_{0} \quad$ :Equivalent radius of the opening (m)

$T_{\infty} \quad$ :Ambient gas temperature (K)

$u_{m} \quad$ :Flow velocity along trajectory $(\mathrm{m} / \mathrm{s})$

$u_{0} \quad$ :Maximum flow velocity at the opening $(\mathrm{m} / \mathrm{s})$

$U_{E} \quad$ :Velocity of flow from the ambient region to the flame region $(\mathrm{m} / \mathrm{s})$

$U_{E, W}$ :Velocity of flow from the wall region to the flame region $(\mathrm{m} / \mathrm{s})$

$V_{E, W} \quad$ :Velocity of flow from the ambient region to the wall region $(\mathrm{m} / \mathrm{s})$

$Z_{N} \quad$ :Height of the neutral plane (m)

$\rho_{\infty} \quad$ :Ambient gas density $\left(\mathrm{kg} / \mathrm{m}^{3}\right)$ 

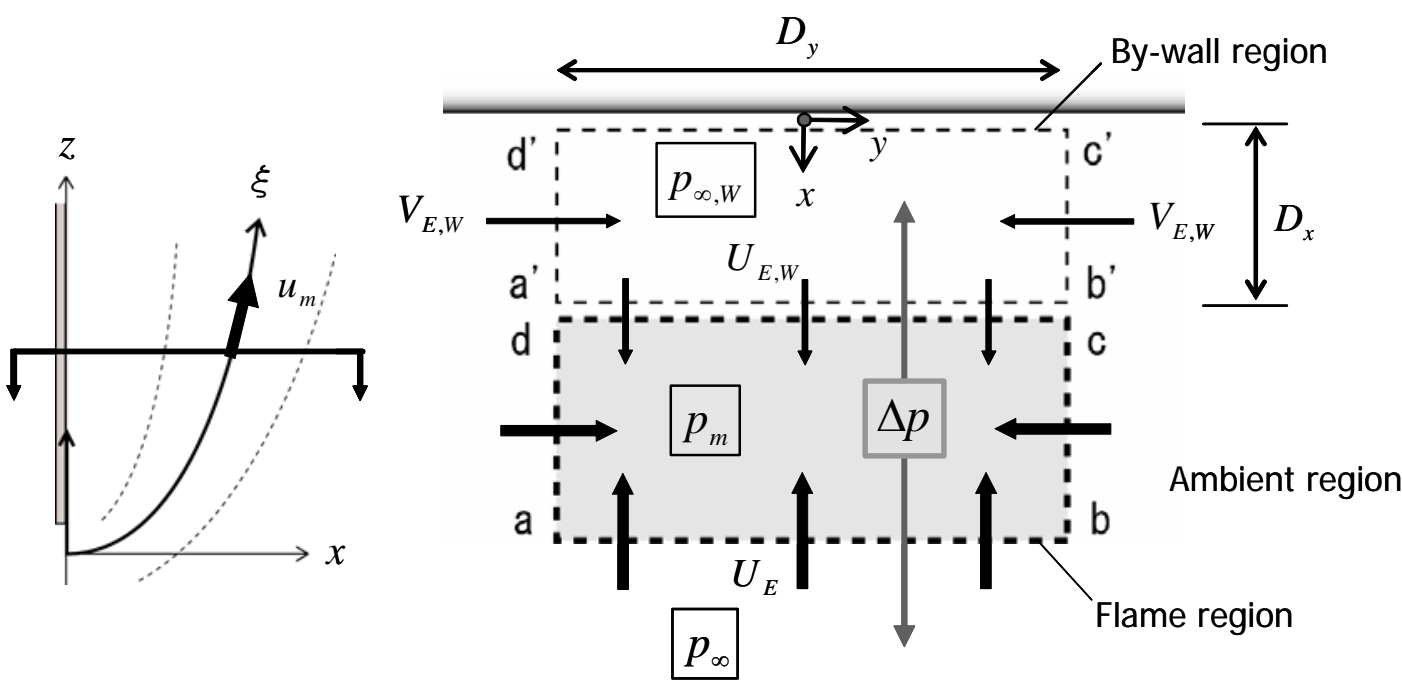

Fig.1. Pressure difference inducing flow around the window flame. 


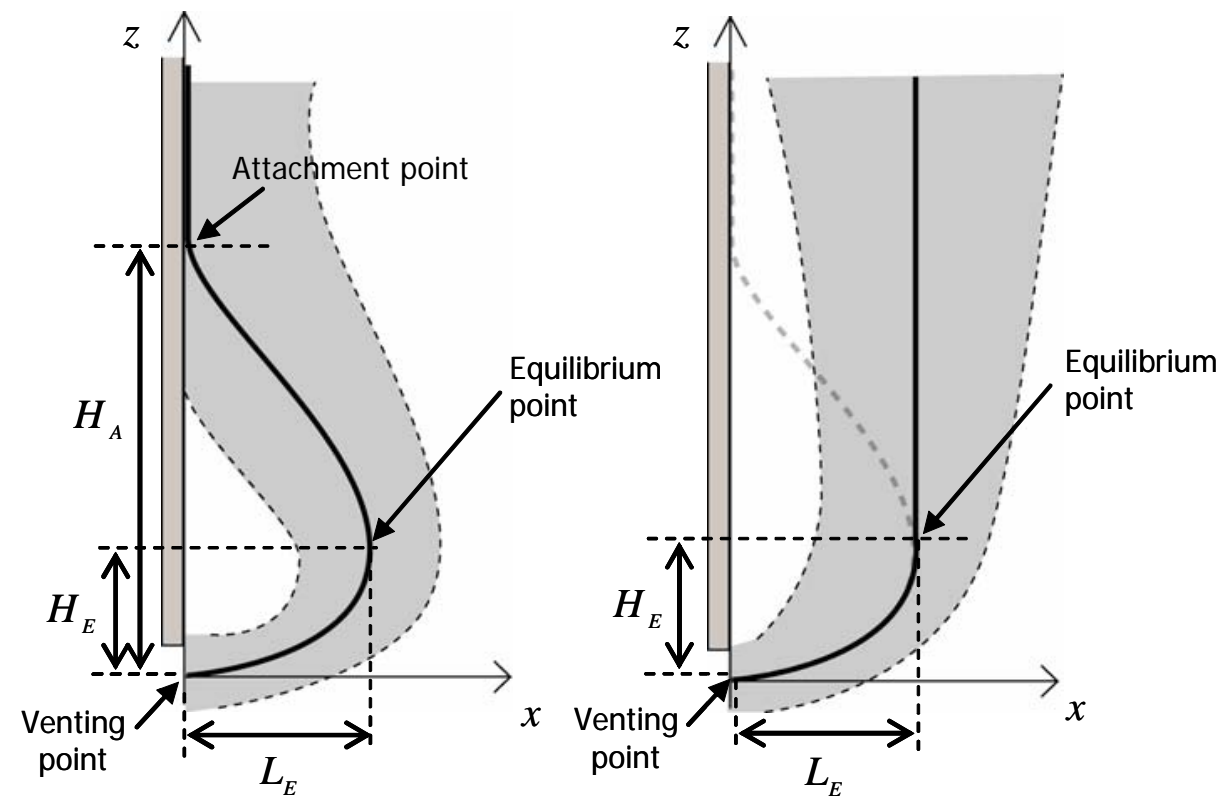

(A) attaching window flame. $\quad$ (B) non-attaching window flame.

Fig.2. Trajectory model for the window flame. 


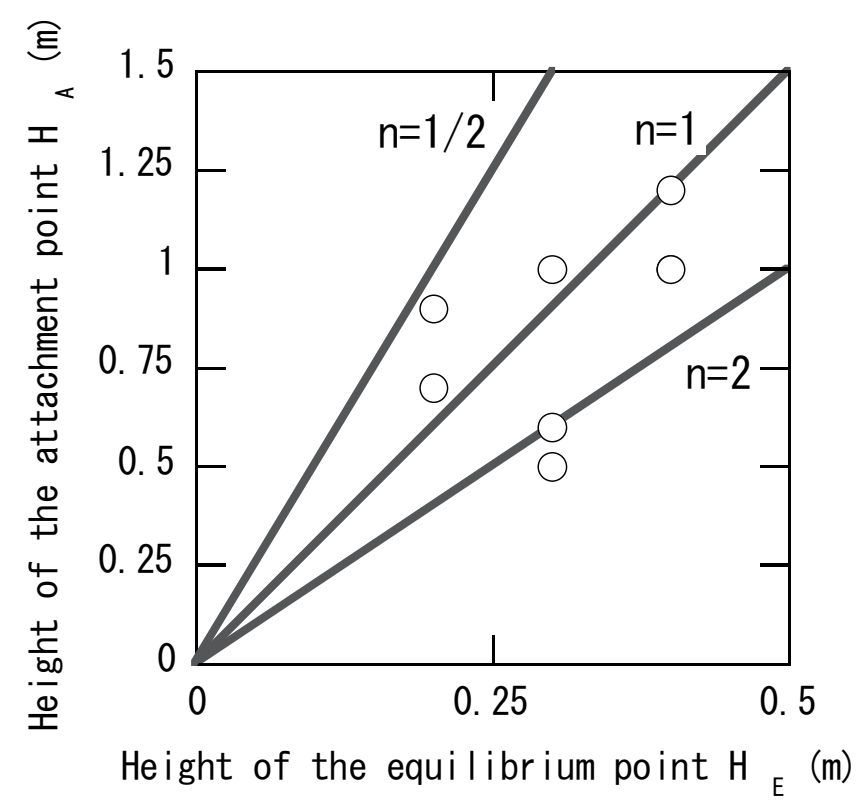

Fig.3. The equilibrium point $H_{E}$ and the attachment point $H_{A}$. 


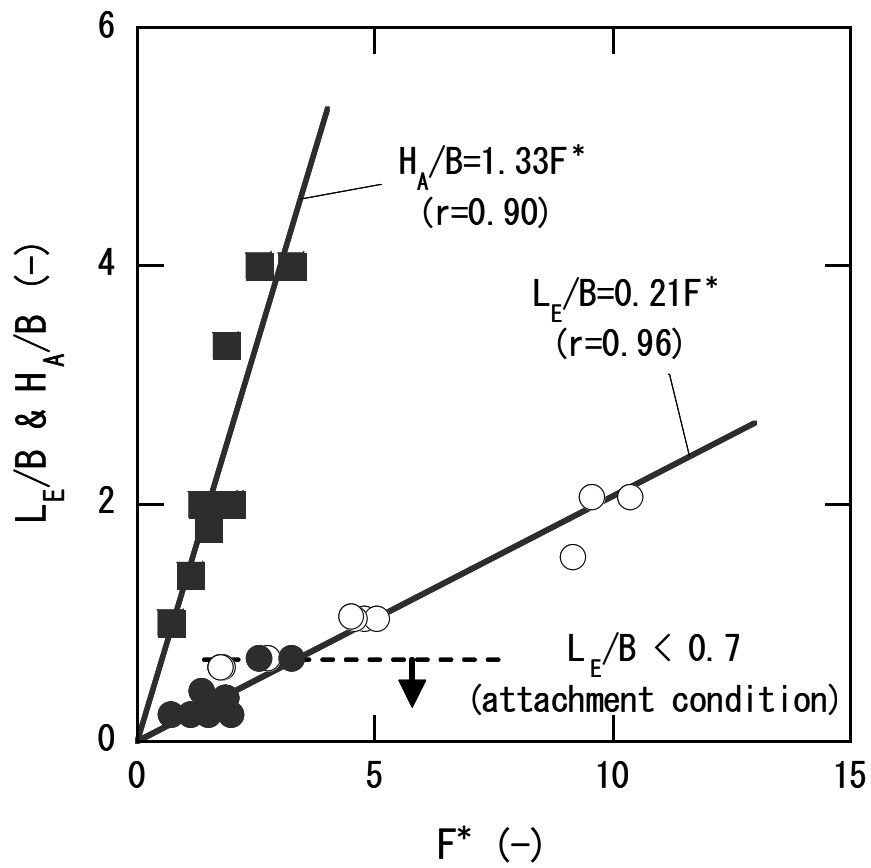

Fig.4. Separations of the equilibrium point from the wall $L_{E}$ and height of the attachment points $H_{A}$. Solid symbols designate the data for the attaching flows. 


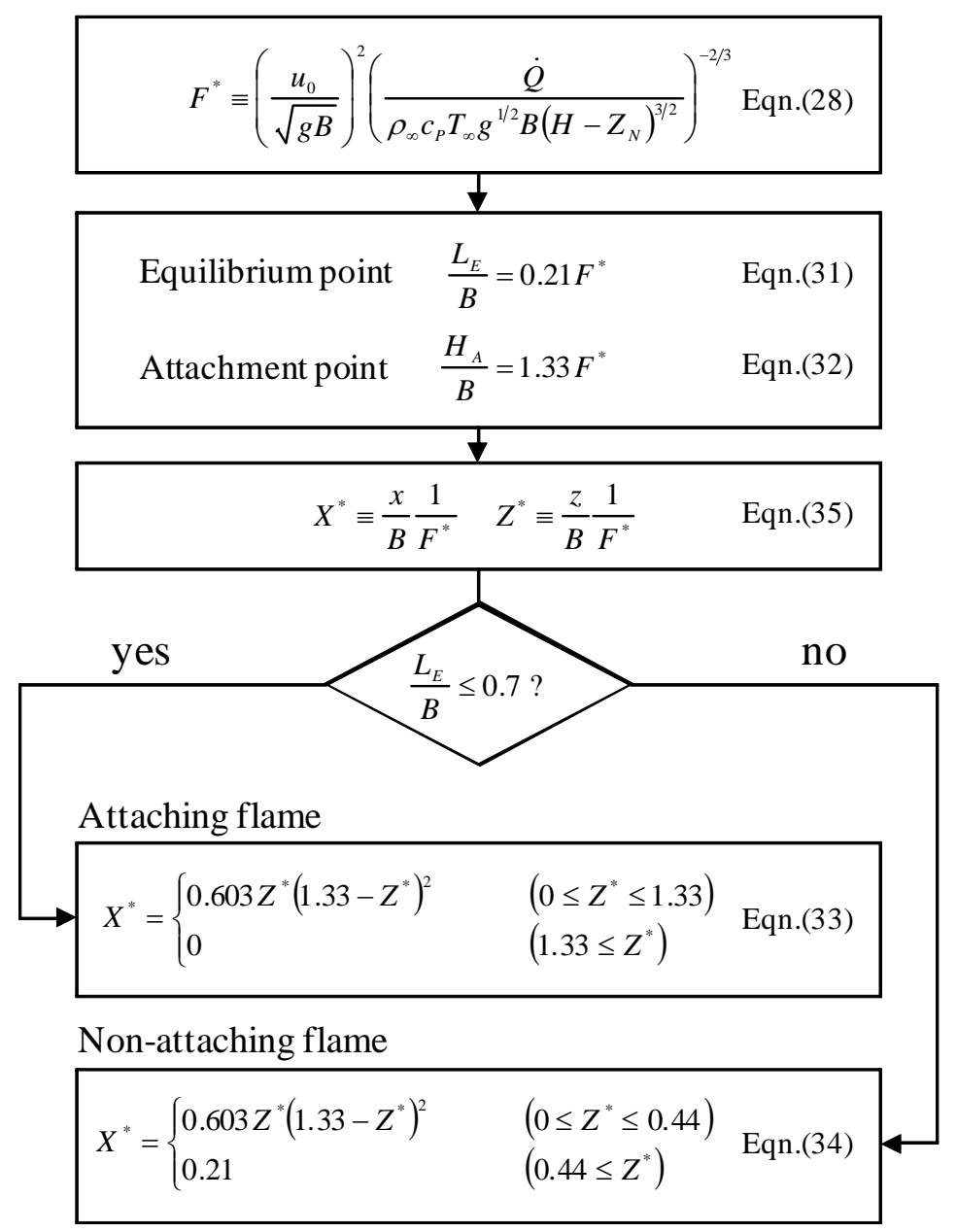

Fig.5. Flowchart of the trajectory calculation. 



(A) $\dot{m}_{0}=0.0 \mathrm{~kg} / \mathrm{s}$.
(B) $\dot{m}_{0}=0.0528 \mathrm{~kg} / \mathrm{s}$.

Fig.6. Trajectory of the window flame $(B=0.2 \mathrm{~m}, \mathrm{H}=0.5 \mathrm{~m})$ 


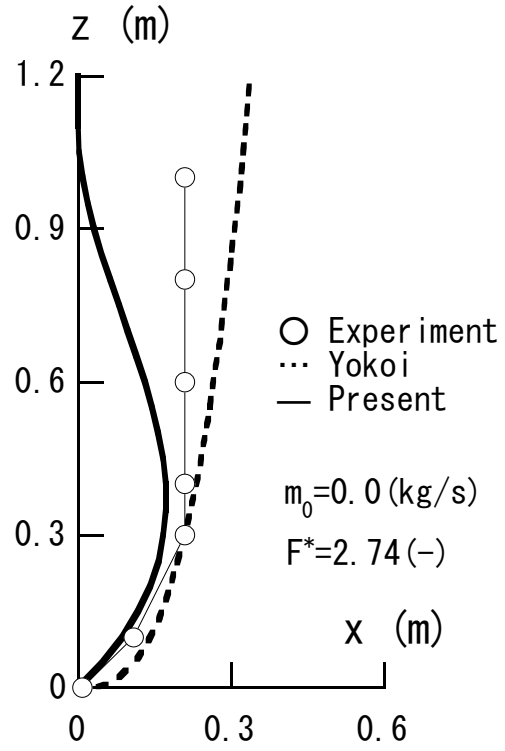

(A) $\dot{m}_{0}=0.0 \mathrm{~kg} / \mathrm{s}$.

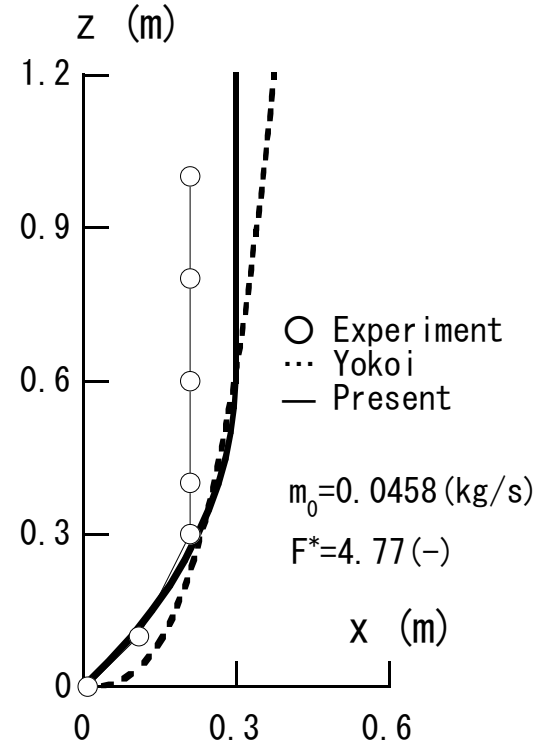

(B) $\dot{m}_{0}=0.0458 \mathrm{~kg} / \mathrm{s}$.

Fig.7. Trajectory of the window flame $(B=0.3 \mathrm{~m}, \mathrm{H}=0.5 \mathrm{~m})$ 


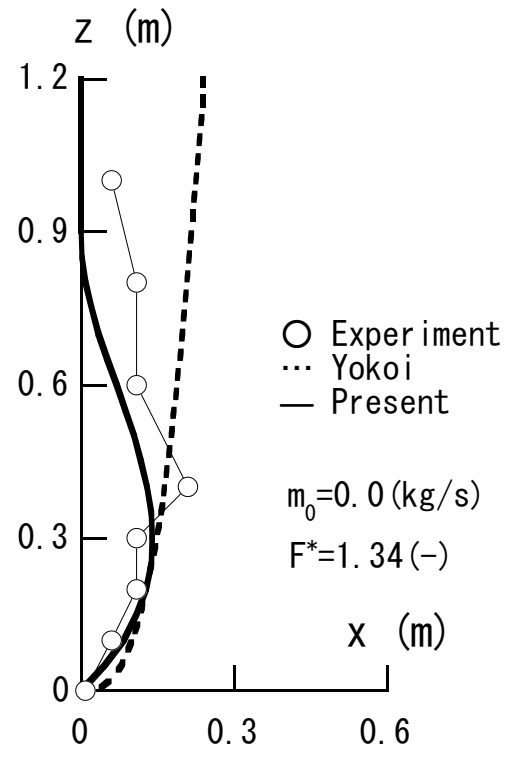

(A) $\dot{m}_{0}=0.0 \mathrm{~kg} / \mathrm{s}$.



(B) $\dot{m}_{0}=0.0548 \mathrm{~kg} / \mathrm{s}$.

Fig.8. Trajectory of the window flame $(B=0.5 \mathrm{~m}, \mathrm{H}=0.5 \mathrm{~m})$ 


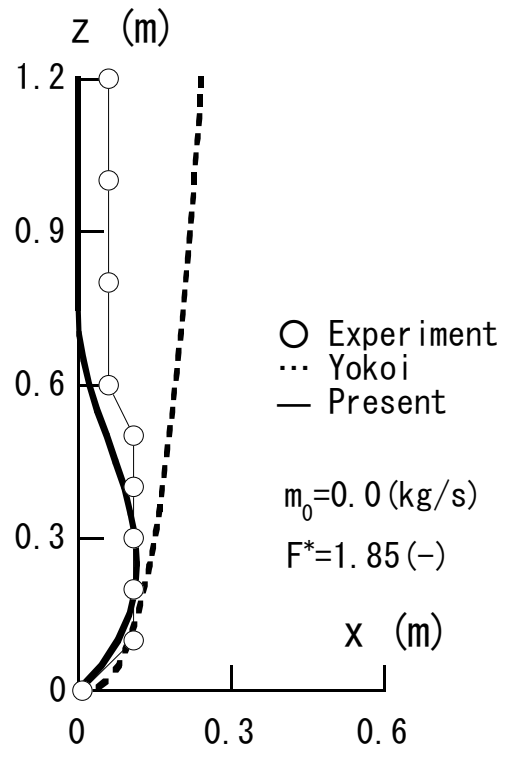

(A) $\dot{m}_{0}=0.0 \mathrm{~kg} / \mathrm{s}$.

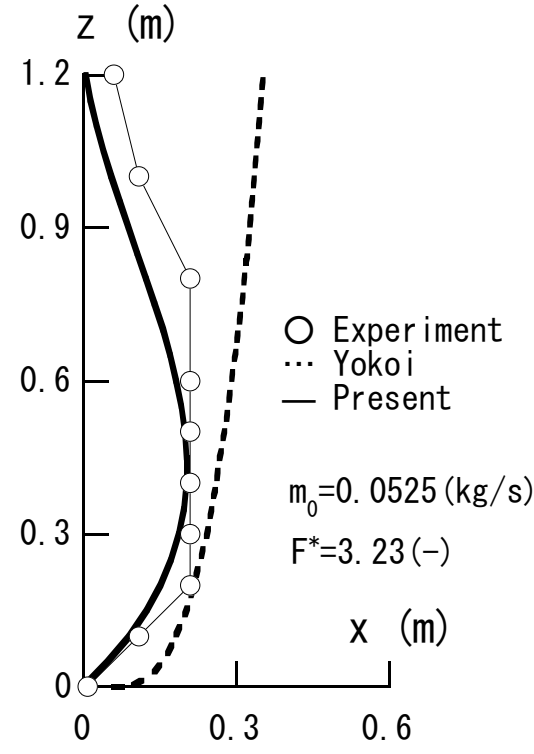

(B) $\dot{m}_{0}=0.0525 \mathrm{~kg} / \mathrm{s}$.

Fig.9. Trajectory of the window flame $(B=0.3 \mathrm{~m}, \mathrm{H}=0.3 \mathrm{~m})$ 


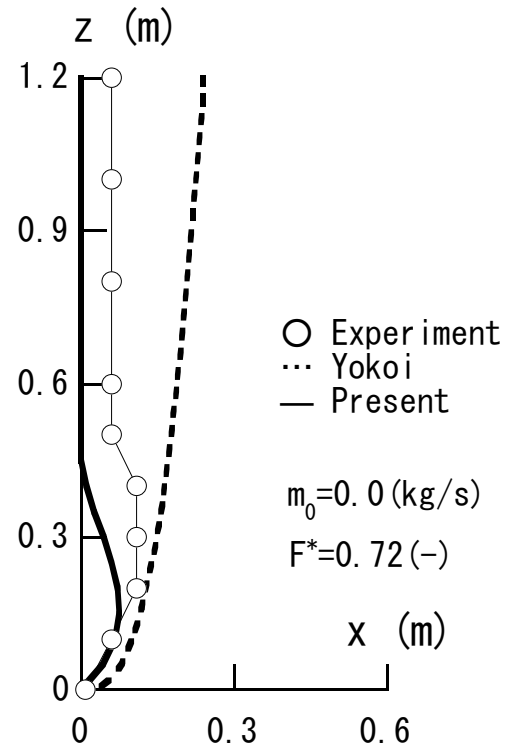

(A) $\dot{m}_{0}=0.0 \mathrm{~kg} / \mathrm{s}$.

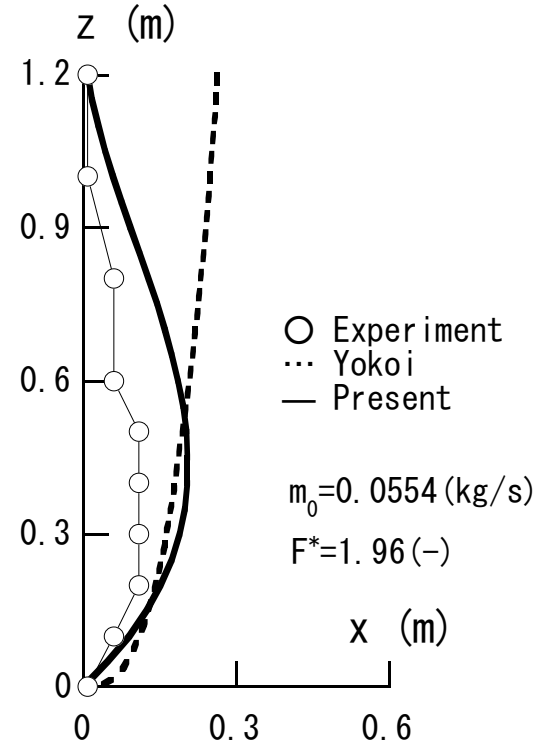

(B) $\dot{m}_{0}=0.0554 \mathrm{~kg} / \mathrm{s}$.

Fig.10. Trajectory of the window flame $(B=0.5 \mathrm{~m}, \mathrm{H}=0.3 \mathrm{~m})$ 